\title{
Ototoxicity of cisplatin administered to guinea pigs via the round window membrane
}

\author{
Li Xia, Zhengnong Chen* and Shankai Yin* \\ Department of Otolaryngology, Affiliated Sixth People's Hospital of Shanghai Jiao Tong University, \\ Otolaryngology Institute of Shanghai Jiao Tong University, 600 Yishan Road, Shanghai 200233, China
}

(Received April 13, 2012; Accepted April 27, 2012)

\begin{abstract}
Animal models of ototoxicity represent an elementary tool in otolaryngologic research. Such models are usually created via the consecutive injection of ototoxic drugs or the co-administration of ethacrynic acid and low-dose ototoxic drugs. Injection via the round window membrane (RWM) is one approach that allows for local drug delivery into the inner ear. In this study, 47 guinea pigs received an injection of varying doses of cisplatin via the RWM, and data concerning the animals' auditory brainstem responses, hair cells, and spiral ganglion neurons were analyzed. Our results indicate the high efficiency and generally small reaction of the subjects, suggesting that the application of cisplatin via the RWM is an effective animal model for ototoxicity research.
\end{abstract}

Key words: Cisplatin, Ototoxicity, Round window membrane, Auditory brainstem response, Hair cell, Spiral ganglion neuron

\section{INTRODUCTION}

Cisplatin is an effective chemotherapeutic drug, but its use is limited because of the risk of ototoxicity, neurotoxicity, and nephrotoxicity (Humes, 1999; Trimmer and Essigmann, 1999; Sastry and Kellie, 2005). The ototoxicity of cisplatin accumulates reactive oxygen species, particularly in the metabolically vigorous outer hair cells (OHCs), spiral ganglion neurons (SGNs), and marginal cells of the stria vascularis (Hamers et al., 2003), then activates a cascade reaction associated with apoptosis (Van De Water et al., 2004). Subsequent death of the OHCs in the basal turn of the basilar membrane leads initially to high-frequency hearing loss that progresses to further cell death throughout the organ of Corti (Alam et al., 2000; Devarajan et al., 2002).

In previous ototoxic studies, cisplatin was used by single or consecutive systemic injection and co-administration of a loop diuretic (Iraz et al., 2005; van Ruijven et al., 2005; Ding et al., 2007). However, severe wholebody reactions leading to a high mortality rate occurred after systemic delivery (Gratton et al., 1990; Tsang et al., 2009), and a high rate of hair cell loss often occurred with the co-administration method which did not provide abun- dant choices of varied levels of hair cell lesion for ototoxic study (Ding et al., 2007). In addition, systemic ototoxic delivery cannot serve as a control for the comparison of hearing function.

Local drug delivery via the round window membrane (RWM) is an alternative method to treat cochlear lesions (He et al., 2009). However, detailed information about hair cell and SGN loss has not been reported. In the present study, the auditory brainstem response (ABR) threshold and the loss of hair cells and SGNs were investigated after cisplatin was injected into the cochlea via the RWM. These parameters were compared with those associated with the ototoxicity of consecutive cisplatin injections.

\section{MATERIALS AND METHODS}

\section{Subjects and groupings}

Forty-seven Dunkin-Hartley guinea pigs with a body weight of 350 to $450 \mathrm{~g}$ were recruited from the Experimental Animal Service of Shanghai Jiaotong University School of Medicine. This study was carried out in strict accordance with the recommendations in the Declaration of Helsinki principles, the protocol was approved by the

\footnotetext{
Correspondence: Zhengnong Chen (E-mail: jassey@126.com)

Shankai Yin (E-mail: yinshankai@china.com)
}

*These authors equally contributed to this work. 
Committee of Experimental Animal Service, Shanghai, China [Permit Number: SYXK (H2011-0128)].

The animals, which were maintained on a $12 \mathrm{hr}$ lightdark cycle during the experiment, were randomly assigned to three groups. Group A $(n=12)$ received consecutive injections of cisplatin (P4394; Sigma-Aldrich, St. Louis, MO, USA) alone ( $5 \mathrm{mg} / \mathrm{kg}$ by intraperitoneal [i.p.] injection per day for three consecutive days) as shown in Table 1. Group B $(n=29)$ received a single injection of cisplatin $(0.5 \mu \mathrm{l})$ via injection into the RWM, and then the animals were divided into three subgroups according to cisplatin concentration (subgroups B1, B2, and B3 for 0.5 , 0.25 , and $0.125 \mathrm{mg} / \mathrm{ml}$, respectively). Group $\mathrm{C}(n=6)$ was the normal control group. The hearing status of the animals was monitored using ABR tests before and seven days after drug administration. After the final functional test, the animals were sacrificed and their cochleae harvested for morphological observation. From the animals treated via the RWM with 0.125 and $0.25 \mathrm{mg} / \mathrm{ml}$ cisplatin (bilateral cisplatin injection) and via consecutive cisplatin injections, one cochlea was used to make a stretched preparation of the basilar membrane to observe lesions in the organ of Corti while the contralateral cochlea was used to make cross-sections of the modiolus to investigate SGN damage. However, of the animals that received treatment via the RWM with $0.5 \mathrm{mg} / \mathrm{ml}$ cisplatin (a unilateral cisplatin injection), the cochlea on the experimental side was used to make both a stretched preparation of the basilar membrane and cross-sections of the modiolus. The contralateral cochlea was used as the control to determine whether cisplatin could travel from one cochlea to the other via the cerebrospinal fluid. All animals were weighed daily between the first and final treatments.

\section{Surgery}

All surgical procedures were performed under a dissecting microscope with sterilized instruments. The animals were anesthetized with $40 \mathrm{mg} / \mathrm{kg}$ ketamine and $8 \mathrm{mg} / \mathrm{kg}$ xylazine (i.p. injection). A random, linear postauricular skin incision was made behind the ear. Blunt dissection through the underlying soft tissue and auditory bulla was then performed. Portions of the skin and nuchal musculature were retracted to expose the posterior aspect of the auditory bulla. A burr hole was made through the bulla using a high-speed dental-type drill and enlarged with forceps until the round window niche was visible. Gelfoam was placed on the surface of the RWM, and $5 \mu \mathrm{l}$ of various cisplatin concentrations was injected using a syringe fitted with a glass needle stabilized with a stereotactic manipulator. The duration of the entire procedure was $\sim 40 \mathrm{~min}$. An analgesic, Butorphanol (Torbugesic ${ }^{\circledR}$, Fort Dodge, IA, USA) $0.1 \mathrm{mg} / \mathrm{kg}$ (i.m.), was injected post-surgically. During the operation, the animals were laid on heat pads until they recovered from the anesthesia; temperature was monitored using a sensor.

\section{ABR tests}

All animals were subjected to closed-field ABR tests using Tucker-Davis Technologies (TDT) hardware and software (Alachua, FL, USA) before treatment to generate control data. A second closed-field ABR test was performed seven days post-surgically. The acoustic stimuli (tone bursts) were synthesized using SigGen software (10 ms duration; $0.5 \mathrm{~ms}$ rise/fall time; Blackman window), amplified by an ES1 amplifier, and transduced by a broadband electrostatic earphone (EC1). The signals were collected by subdermal electrodes and amplified 20 times; band-pass filters were set between $100 \mathrm{~Hz}$ and $3 \mathrm{kHz}$. The

Table 1. Hair cell loss (\%) by group and anatomical location

\begin{tabular}{|c|c|c|c|c|c|c|c|}
\hline Subgroup & $\mathrm{n}$ & $\mathrm{HC}$ & $1^{\text {st }}$ turn & $2^{\text {nd }}$ turn & $3^{\text {rd }}$ turn & $4^{\text {th }}$ turn & Total \\
\hline \multirow{2}{*}{ Group A } & \multirow{2}{*}{7} & $\mathrm{IHC}$ & 0 & 0 & 0 & 0 & 0 \\
\hline & & $\mathrm{OHC}$ & $55.8 \pm 9.8 \%$ & $23.2 \pm 8.1 \%$ & $6.7 \pm 2.3 \%$ & $4.0 \pm 0.7 \%$ & $30.9 \pm 3.6 \%$ \\
\hline \multirow{3}{*}{ Subgroup B1 } & \multirow{3}{*}{7} & $\mathrm{IHC}$ & $19.3 \pm 5.6 \%$ & $1.7 \pm 0.7 \%$ & 0 & 0 & $8.1 \pm 3.1 \%$ \\
\hline & & $\mathrm{OHC}$ & $96.7 \pm 2.2 \%$ & $50.9 \pm 5.2 \%$ & $22.2 \pm 1.6 \%$ & $16.9 \pm 0.4 \%$ & $59.9 \pm 4.5 \%$ \\
\hline & & & $0.17 \pm 0.2 \%$ & & & & $0.07 \pm 0.1 \%$ \\
\hline \multirow{2}{*}{ Subgroup B2 } & \multirow{2}{*}{6} & $\mathrm{IHC}$ & $4.5 \pm 3.8 \%$ & 0 & 0 & 0 & $0.8 \pm 0.7 \%$ \\
\hline & & $\mathrm{OHC}$ & $28.2 \pm 5.5 \%$ & $6.7 \pm 1.1 \%$ & $2.4 \pm 0.2 \%$ & $1.1 \pm 0.1 \%$ & $13.4 \pm 3.1 \%$ \\
\hline \multirow{2}{*}{ Subgroup B3 } & \multirow{2}{*}{6} & $\mathrm{IHC}$ & 0 & 0 & 0 & 0 & 0 \\
\hline & & $\mathrm{OHC}$ & $0.17 \pm 0.2 \%$ & 0 & 0 & 0 & $0.07 \pm 0.1 \%$ \\
\hline
\end{tabular}

The percentage was calculated as the result of lost hair cells in the corresponding region. $\mathrm{n}=$ sample size. 
Ototoxicity of cisplatin via round window membrane

signals were input to a real-time processor (RA16BA), processed using BioSig software (TDT), and averaged for 1,000 sweeps in each trial. The threshold was evaluated at 1, 2, 4, 8, 16, and $32 \mathrm{kHz}$. At each frequency, the stimuli were presented at $90 \mathrm{~dB}$, decreased in $5 \mathrm{~dB}$ steps until the threshold was approached, and then decreased in $5 \mathrm{~dB}$ steps until the ABR wave disappeared. The threshold was defined as the lowest intensity at which a visible and repeatable ABR wave was observed in two averaged runs. The body temperature of the animals was maintained at $38^{\circ} \mathrm{C}$ with a thermostatic heating pad.

\section{Stretched preparation of the basilar membrane}

After the final ABR test, the animals were euthanized with an overdose of pentobarbital $(200 \mathrm{mg} / \mathrm{kg}$, i.p. injection), and their temporal bones were harvested. Each bulla was opened using rongeurs to expose the cochlea. The oval window and round window were then opened. Following the creation of a hole in the cochlear apex, $4 \%$ paraformaldehyde in $0.1 \mathrm{M}$ phosphate-buffered saline (PBS, pH 7.4) was perfused through the cochlea for at least $4 \mathrm{hr}$. The basilar membrane was dissected under a dissecting microscope, and the stria vascularis and tectorial membrane were removed. To identify F-actin in the organ of Corti, rhodamine phalloidin $(5 \mu \mathrm{g} / \mathrm{ml}$; P1951; Sigma-Aldrich; St. Louis, MO, USA) was applied for $20 \mathrm{~min}$ at room temperature away from light. The specimens were then rinsed three times with $0.1 \mathrm{M}$ PBS. The fluorescent signals of the hair cells were counted under a Nikon light microscope with epifluorescence and a $20 \times$ objective.

\section{Semi-thin sections}

The cochleas were fixed with $2.5 \%$ glutaric dialdehyde for $4 \mathrm{~h}$ at room temperature. Following several rinses in $0.1 \mathrm{M}$ sodium cacodylate buffer ( $\mathrm{pH} 7.4$ ), the modiolus was decalcified in $10 \%$ EDTA on a swing bed for five days at room temperature. Next, the specimens were post-fixed in $1 \% \mathrm{OsO}_{4}$ in $0.1 \mathrm{M}$ sodium cacodylate buffer ( $\mathrm{pH} \mathrm{7.4)} \mathrm{for} 2 \mathrm{hr}$ at $4^{\circ} \mathrm{C}$. Dehydration was performed in a graded ethanol-propylene oxide series with ethoxyline resin. The modioli were embedded in the same resin overnight at $70^{\circ} \mathrm{C}$. For light microscopic assessment of cochlear pathologies, semi-thin $(1 \mu \mathrm{m})$ sections in the mid-modiolar plane were cut with diamond knives, collected on glass slides, stained with $1 \%$ methylene blue, and rinsed with distilled water three times. The sections were then examined and photographed with a Nikon light microscope for qualitative and quantitative analyses of the SGNs in Rosenthal's canal from the first to the third turn. At least $10 \mathrm{SGN}$ slices were counted per sample.

\section{Statistics}

The SPSS statistical software, version 18.0 (SPSS Inc., Chicago, IL, USA) was used for statistical analysis. Threshold shifts, cell loss, and cell survival across the groups and subgroups were analyzed using one-way repeated measures (RM) analysis of variance (ANOVA) or two-way RM ANOVA. A difference was deemed to be significant at $p<0.05$. All data are presented as the mean \pm standard error of the mean (S.E.M.).
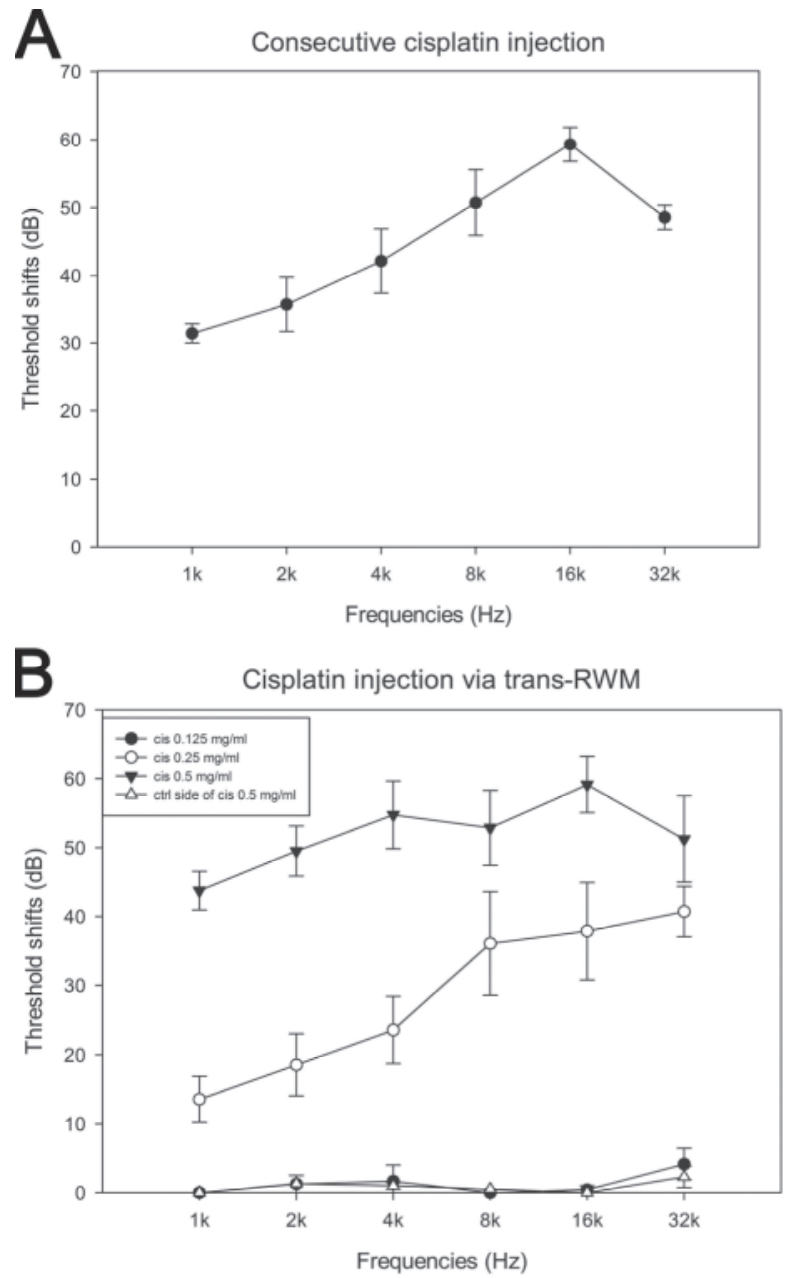

Fig. 1. Threshold shifts of whole frequencies against the baseline. (A) Threshold shifts across frequencies seven days post-consecutive cisplatin application (Group A). Larger shifts were observed at higher frequencies (16 and $32 \mathrm{kHz}$ ). (B) Threshold elevation seven days after the injection of cisplatin at various concentrations via the RWM; $0.5 \mathrm{mg} / \mathrm{ml}$ cisplatin caused the largest shifts across frequencies, and there were no significant shifts in the $0.125 \mathrm{mg} / \mathrm{ml}$ cisplatin and contralateral application of $0.5 \mathrm{mg} / \mathrm{ml}$ cisplatin groups. 


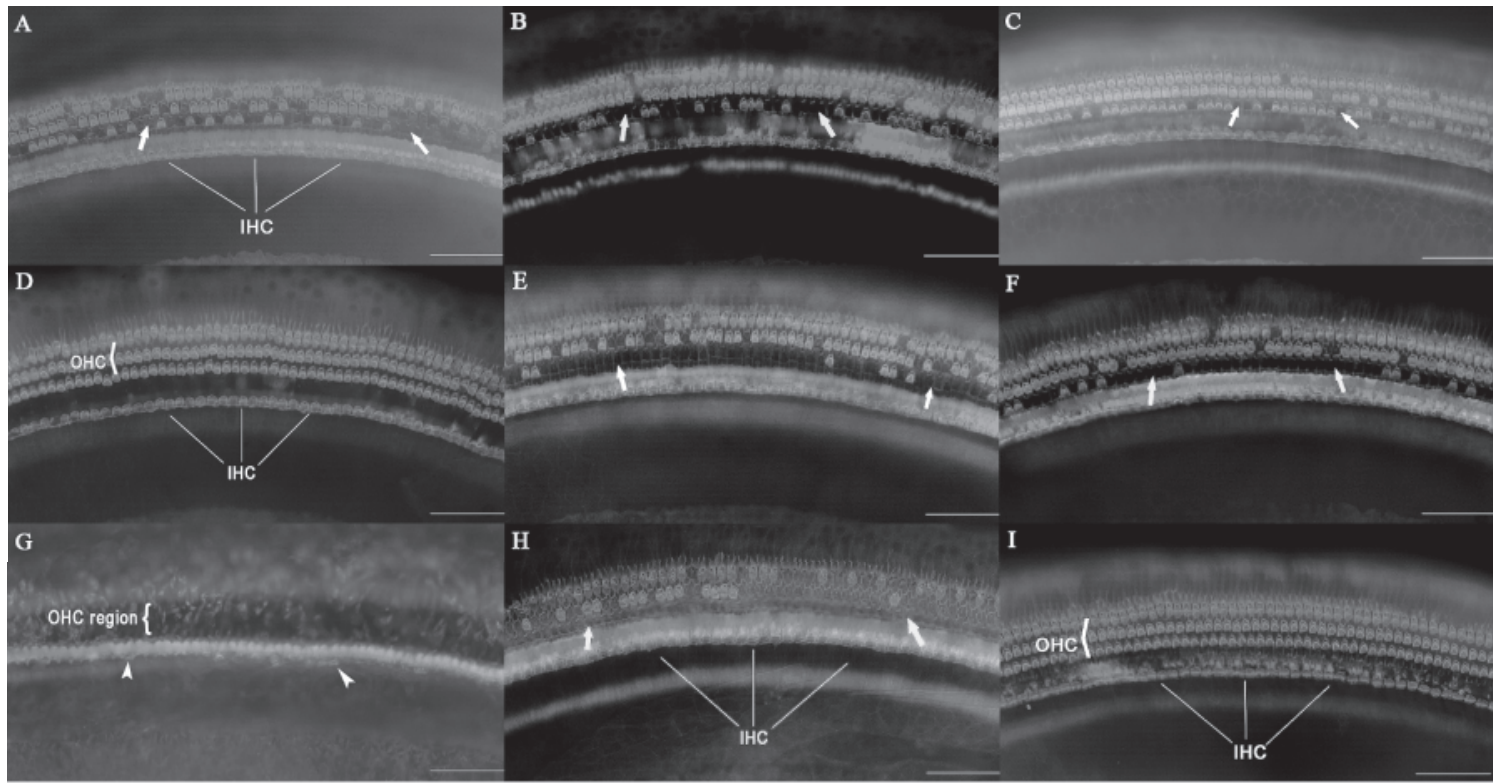

Fig. 2. Representative images of the surface preparation from various treatments. Consecutive cisplatin injection $(5 \mathrm{mg} / \mathrm{kg}$ for three days) causes more severe OHC loss in the basal turn (A), while fewer lesions occur in the apical turn (B). Application of $0.125 \mathrm{mg} / \mathrm{ml}$ cisplatin via the RWM (Subgroup B3) causes sparse OHC loss in the basal turn (C) and non-significant OHC loss in the apical turn (D). OHC loss is observed in the basal turn (E) and apical turn (F) with $0.25 \mathrm{mg} / \mathrm{ml}$ cisplatin treatment via the RWM (Subgroup B2). Massive OHC loss with IHC loss is observed in the basal turn in Subgroup B1 (G); severe OHC loss is observed in the apical turn $(\mathrm{H})$. The hair cells remain intact on the side contralateral to the $0.5 \mathrm{mg} / \mathrm{ml}$ cisplatin-treated ear (I). The arrow points to the location at which OHCs are lost. The arrowhead points to a surviving IHC (G). Scale bar: $50 \mu \mathrm{m}$

\section{RESULTS}

\section{Effect of cisplatin on hearing function}

The ABR threshold was elevated across all frequencies seven days after the final cisplatin injection in the systemic group (Group A). Compared with the baseline, there was significant threshold elevation across all frequencies, with an average of $42 \mathrm{~dB}$. More hearing loss was found at higher frequencies with the largest shift at $16 \mathrm{kHz}(59 \pm$ $2.5 \mathrm{~dB}$ ), as shown in Fig. 1A.

Cisplatin injections via the RWM also caused hearing loss in guinea pigs. However, almost no threshold shifts were observed at each frequency in Subgroup B3. The thresholds across frequencies were elevated in both Subgroups B1 and B2. A greater threshold shift was detected in the group that received a higher cisplatin concentration. For example, in Subgroup B1, a $40 \mathrm{~dB}$ shift was observed on average, while in Subgroup B2, a $23 \mathrm{~dB}$ threshold shift was revealed (Fig. 1B). A statistically significant difference in ABR shifts was found among these three groups $(p<0.01$ effect of frequency and dose, twoway RM ANOVA).

\section{Effect of cisplatin on the organ of Corti}

Hair cell loss was found in the cochleae of the systemic delivery group. Consistent with the functional results, the hair cell lesions were more severe at the basal turn and gradually diminished toward the apex. It was evident that damage to cochlear hair cells was greater for OHCs than IHCs (Figs. 2A, 2B and 3A). Almost no IHC loss was found in this group.

Similar to the effects of systemic application of cisplatin, lesions in the organ of Corti were also exhibited after cisplatin administration via the RWM. OHC losses were found in all three cisplatin local application subgroups, with the most severe lesions in highest-dose subgroups (Figs. 2G and H). OHC losses in the three subgroups were most severe in the basal turn (Figs. 2C-H). IHC losses were also demonstrated in the local delivery group. However, only $0.5 \mathrm{mg} / \mathrm{ml}$ cisplatin caused apparent IHC lesions (Fig. 2G). No hair cell loss was found in the contralateral ear of B1 subgroup (Fig. 2I).

The cochleogram for Group A is shown in Fig. 3A. The average $\mathrm{OHC}$ loss was $31 \%$, but no significant IHC losses were seen. For local injection, the average $\mathrm{OHC}$ loss was $60 \%$ in Subgroup B1, 13\% in Subgroup B2, and 0.1\% 
Ototoxicity of cisplatin via round window membrane
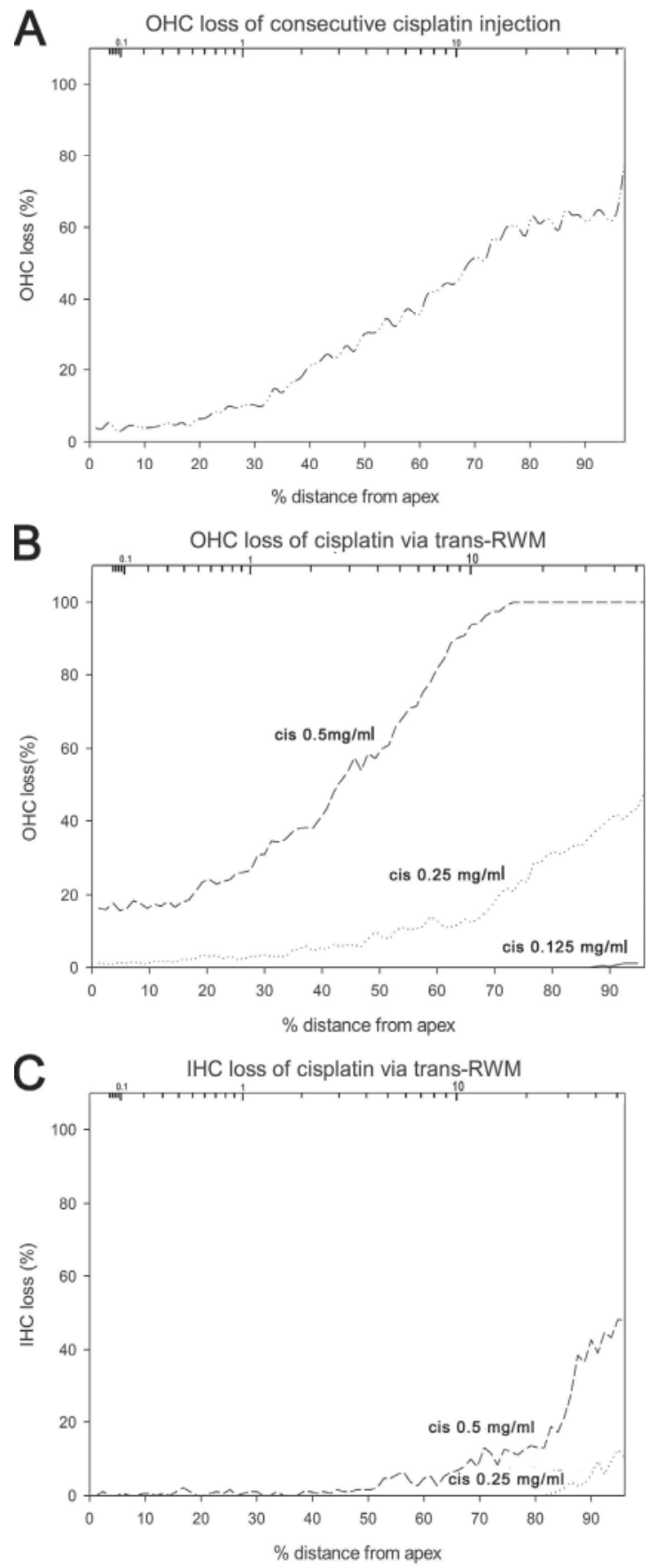

Fig. 3. Cochleogram indicating hair cell loss. OHCs were counted at 10 locations along the basilar membrane from apex to base (lower horizontal axis). The upper horizontal axis was the frequency-place map (Greenwood, 1990). (A) OHC loss in Group A. (B) $\mathrm{OHC}$ loss in the subgroups that received cisplatin via the RWM. OHC in the basal turn encountered nearly $100 \%$ loss. (C) IHC loss in B1 and B2 subgroups. There was sparse loss of IHC in B2 subgroup. in Subgroup B3 (Fig. 3B and Table 1). The OHC losses in these three subgroups were statistically significant ( $p<0.01$, one-way RM ANOVA). Meanwhile, the average IHC loss was $8 \%$ in Subgroup B1, 0.8\% in Subgroup B2, and 0\% in Subgroup B3 (Fig. 3C and Table 1).

\section{Effect of cisplatin on SGNs}

In guinea pigs that received consecutive cisplatin applications, SGN lesions in the basal turn were observed as myelin sheath detachment from the perikarya (Fig. 4B). However, no decrease in SGN density was observed under this condition.

For the local application groups, the lesions of the SGNs in the two low-dose subgroups (B2 and B3) were tender, but showed no significant abnormalities in quantity (Fig. 5) or quality (Figs. 4C and D). Serious SGN lesions were observed in the basal turn in B1 cisplatin subgroup (Fig. 4E). No significant SGN lesion was observed in the apical turn.

The percent survival of SGNs was compared with the control value, which was set at $100 \%$ (Fig. 5). Both of systemic delivery (Group A) and local application of $0.5 \mathrm{mg} / \mathrm{ml}$ cisplatin (Group B1) caused significant SGN lesions in the basal turn ( $t$-tests, $p<0.05$ for the counts of normal SGNs in basal turn of each group compared with those of control group).

\section{Body weight and mortality}

After receiving the cisplatin treatment, all animals exhibited body weight loss. However, the body weight of the groups treated with cisplatin via the RWM recovered seven days post-surgically; a progressive and severe decrease in body weight was observed in the animals that received consecutive cisplatin injections (Fig. 6).

Of 12 guinea pigs that received consecutive cisplatin injections in the systemic delivery group, seven survived, producing a mortality rate of $42 \%$. Of the 29 guinea pigs that received cisplatin injections via the RWM, 26 survived for seven days post-injection. Cisplatin application via the RWM produced a $10 \%$ mortality rate versus $42 \%$ for consecutive cisplatin administration, revealing an advantage in local drug application. A significant difference in mortality between the two groups was detected ( $p<0.05$, chi-square analysis).

\section{DISCUSSION}

In the present study, ototoxicity caused by cisplatin application directly to the RWM was investigated in guinea pigs. Similar to systemic delivery, cisplatin injection via the RWM could also cause significant ABR threshold 


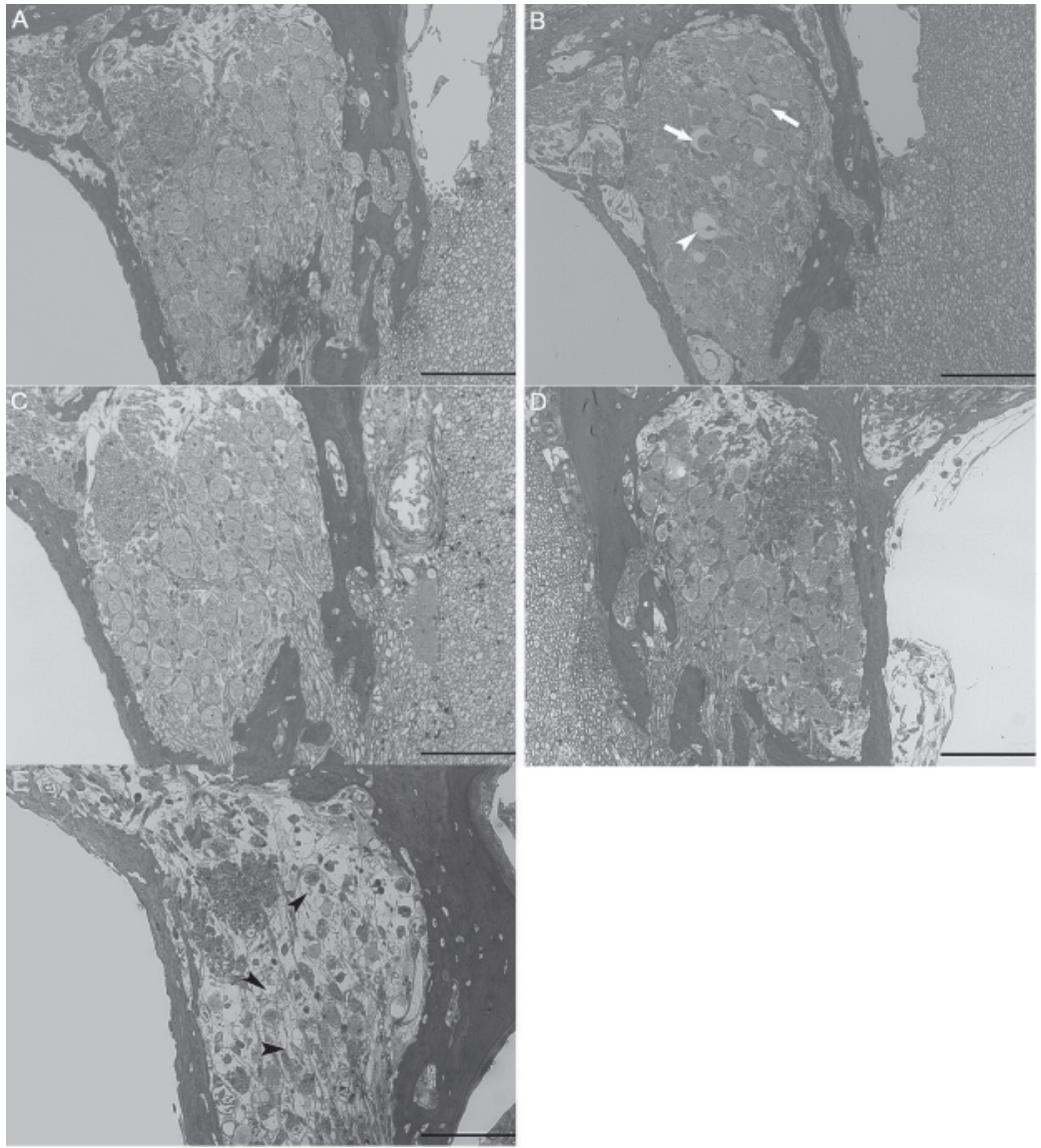

Fig. 4. Representative images of SGNs in cross-section. (A) Normal control. Consecutive cisplatin application causes myelin sheath detachment (arrow) and cell death (arrowhead) in some SGNs in the basal turn of Rosenthal's canal (B). No significant SGN lesions are observed in B2 and B3 subgroups (C and D). Severe SGN loss occurs in B1 subgroup, as shown in (E). Scale bar: $50 \mu \mathrm{m}$.

shifts as well as hair cell and SGN losses.

Cisplatin injection via the RWM offers several advantages compared with systemic delivery. First, a small amount of drug is used locally, resulting in less adverse effects on the animals. Smaller body weight decreases and lower mortality in guinea pigs treated with cisplatin via the RWM confirmed this assertion. The temporary decrease in weight observed post-surgically in this study was caused by anesthesia and pain because no relationship was found between the concentration of cisplatin in the perilymph and weight (Sepmeijer and Klis, 2009). Second, each animal can be used as its own control. No threshold shift and no hair cell or SGN loss was noted at the highest cisplatin concentration $(0.5 \mathrm{mg} / \mathrm{ml})$ for treatment of the contralateral cochlea, such concentration is the highest solubility of cisplatin in normal saline
(Hincal et al., 1979).

Compared with other local delivery methods to the cochlea (i.e., intracochlear injection by cochleostomy), delivery via the RWM has its own advantages. First, the procedure is simple and repeatable. Second, cochlear function (e.g., compound action potentials) can be tested directly in subsequent experiments. Third, fewer histopathologic and anatomical changes following delivery via the RWM were noted compared with cochleastomy (Dimitrov and Duckert, 1985).

Cisplatin delivered via the RWM produced a higher efficiency of ototoxicity in cochlear cells than did cisplatin delivered systemically. The agent enters the scala tympani, directly affecting OHCs, and subsequently approaches Rosenthal's canal through the habenula perforata to affect SGNs by local injection via the RWM (Fritzsch et al., 


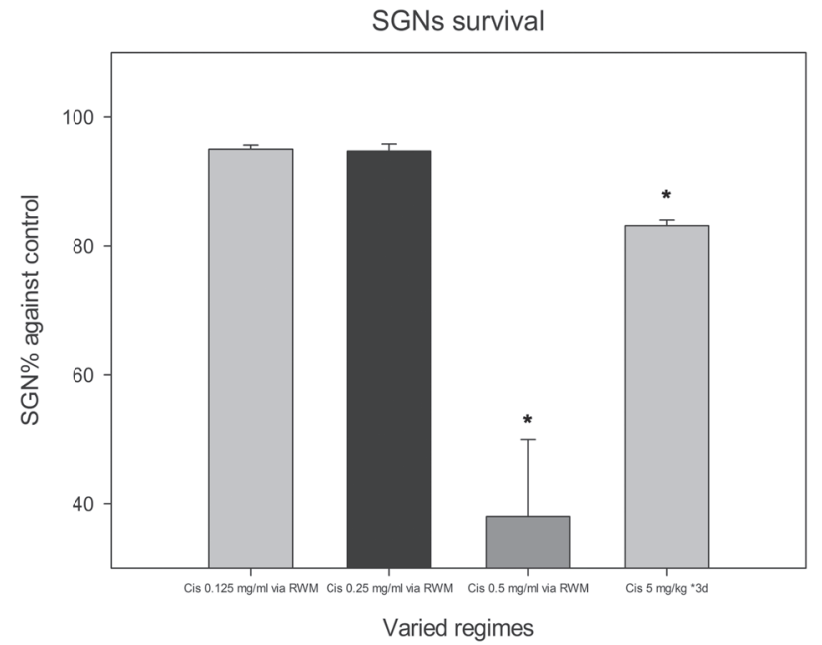

Fig. 5. SGN lesions at basal turn across the groups, using control animal values as $100 \%$. Significant reduction of $\% \mathrm{SGN}$ was observed only in the basal turn in the Groups A and Subgroup B1 $(p<0.05$ indicated by *).

1997). Increasing the dose of cisplatin directly increases its concentration in the perilymph, resulting in greater hair cell loss. OHCs encounter severe loss due to large dose of cisplatin breaking into perilymph followed by increase of reactive oxygen species (Hamers et al., 2003). Large amount of OHC loss leads to perforation of the cuticular plate in OHC (Ramirez-Camacho et al., 2004). Through such perforations, endolymph with potassium ion of high concentration enters perilymph, causing potassium ioninduced apoptosis in IHCs (Ding and Salvi, 2005). In our study, it was shown that only high dose of cisplatin (subgroup of $0.5 \mathrm{mg} / \mathrm{ml}$ ) which brought about swift and severe OHC lesion could cause apparent IHC loss in just 7 days post application. The regimen of cisplatin via trans-RWM caused violent damage of SGNs, just one week after drug treatment, approximate $60 \%$ SGNs in basal turn encountered lesion compared with approximate $20 \%$ SGNs with myelin sheath detachment other than loss via the regimen of consecutive injections. It may cost at least five weeks that apparent SGNs density decreased after the consecutive cisplatin injections (Poirrier et al., 2010).

The pathway of trans-RWM differs from that produced by systemic administration: the drug enters the cochlea through the blood-labyrinth barrier (Laurell et al., 1995, Sepmeijer and Klis, 2009). Such a barrier between the plasma and perilymph can partly explain the lower efficiency of ototoxicity via systemic administration. Although increasing the dose can directly enhance the

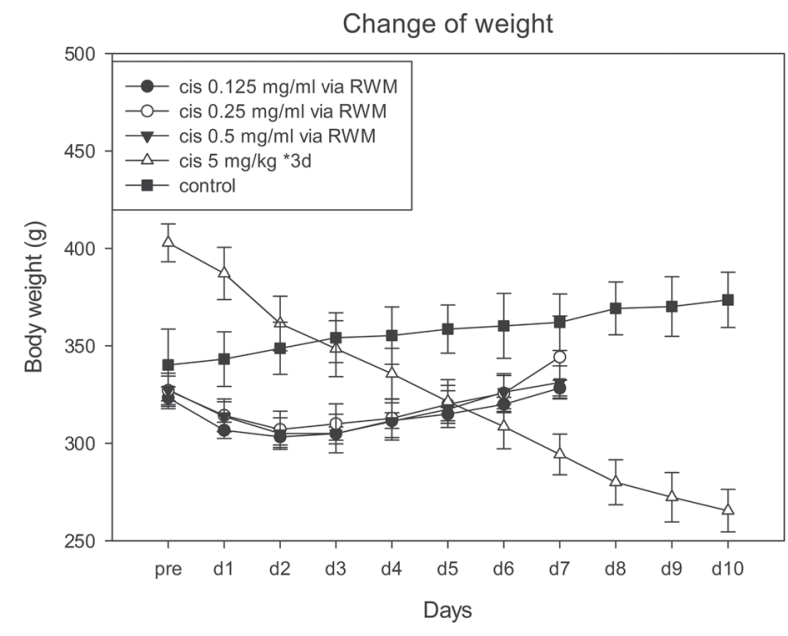

Fig. 6. Body weight changes. The curve for consecutive cisplatin injections showed progressive decrease of body weight since the drug was applied, the curves representing for local cisplatin injection performed slight decrease of body weight followed by an increase 3 days post surgery while the curve for normal group exhibited a stable rise of body weight during such period.

Key Development Program for Basic Research of China (Grant No. 2011CB504503), the National Science Foundation for Distinguished Young Scholars of China (Grant No. 30925035), the National Natural Science Foundation of China (Grant No. 81170920), and the National Natural Science Foundation of China (Grant No. 30901669).

\section{REFERENCES}

Alam, S.A., Ikeda, K., Oshima, T., Suzuki, M., Kawase, T., Kikuchi, T. and Takasaka, T. (2000): Cisplatin-induced apoptotic cell death in Mongolian gerbil cochlea. Hear Res., 141, 28-38.

Devarajan, P., Savoca, M., Castaneda, M.P., Park, M.S., Esteban-Cruciani, N., Kalinec, G. and Kalinec, F. (2002): Cisplatin-induced apoptosis in auditory cells: role of death receptor and mitochondrial pathways. Hear Res., 174, 45-54.

Dimitrov, E.A. and Duckert, L.G. (1985): Morphologic changes in the guinea pig cochlea following cochleostomy--a preliminary scanning electron microscope study. Otolaryngol Head Neck Surg., 93, 408-413.

Ding, D., Jiang, H., Wang, P. and Salvi, R. (2007): Cell death after co-administration of cisplatin and ethacrynic acid. Hear Res. 226, 129-139.

Ding, D. and Salvi, R. (2005): Review of Cellular Changes in the Cochlea Due to Aminoglycoside Antibiotics. Volta Review., 105, 407-438.

Fritzsch, B., Farinas, I. and Reichardt, L.F. (1997): Lack of neurotrophin 3 causes losses of both classes of spiral ganglion neurons in the cochlea in a region-specific fashion. J. Neurosci., 17, 6213-6225.

Gratton, M.A., Salvi, R.J., Kamen, B.A. and Saunders, S.S. (1990): 
L. Xia et al.

Interaction of cisplatin and noise on the peripheral auditory system. Hear Res., 50, 211-223.

Greenwood, D.D. (1990): A cochlear frequency-position function for several species--29 years later. J. Acoust. Soc. Am., 87, 2592-2605.

Hamers, F.P., Wijbenga, J., Wolters, F.L., Klis, S.F., Sluyter, S. and Smoorenburg, G.F. (2003): Cisplatin ototoxicity involves organ of Corti, stria vascularis and spiral ganglion: modulation by alphaMSH and ORG 2766. Audiol. Neurootol., 8, 305-315.

He, J.,Yin, S.,Wang, J., Ding, D. and Jiang, H. (2009): Effectiveness of different approaches for establishing cisplatin-induced cochlear lesions in mice. Acta Otolaryngol., 129, 1359-1367.

Hincal, A.A., Long, D.F. and Repta, A.J. (1979): Cis-platin stability in aqueous parenteral vehicles. J. Parenter. Drug Assoc., 33, 107-116.

Humes, H.D. (1999): Insights into ototoxicity. Analogies to nephrotoxicity. Ann. N Y Acad. Sci., 884, 15-18.

Iraz, M., Kalcioglu, M.T., Kizilay, A. and Karatas, E. (2005): Aminoguanidine prevents ototoxicity induced by cisplatin in rats. Ann. Clin. Lab. Sci., 35, 329-335.

Laurell, G., Andersson, A., Engstrom, B. and Ehrsson, H. (1995): Distribution of cisplatin in perilymph and cerebrospinal fluid after intravenous administration in the guinea pig. Cancer Chemother Pharmacol., 36, 83-86.

Poirrier, A.L.,Van den Ackerveken, P., Kim, T.S., Vandenbosch, R., Nguyen, L., Lefebvre, P.P. and Malgrange, B. (2010): Ototox- ic drugs: difference in sensitivity between mice and guinea pigs. Toxicol. Lett., 193, 41-49.

Ramirez-Camacho, R., Garcia-Berrocal, J.R., Bujan, J., Martin-Marero, A. and Trinidad, A. (2004): Supporting cells as a target of cisplatin-induced inner ear damage: therapeutic implications. Laryngoscope, 114, 533-537.

Sastry, J. and Kellie, S.J. (2005): Severe neurotoxicity, ototoxicity and nephrotoxicity following high-dose cisplatin and amifostine. Pediatr Hematol Oncol., 22, 441-445.

Sepmeijer, J.W. and Klis, S.F. (2009): Distribution of platinum in blood and perilymph in relation to cisplatin induced ototoxicity in the guinea pig. Hear Res. 247, 34-39.

Trimmer, E.E. and Essigmann, J.M. (1999): Cisplatin. Essays Biochem., 34, 191-211.

Tsang, R.Y.,Al-Fayea, T. and Au, H.J. (2009): Cisplatin overdose: toxicities and management. Drug Saf., 32, 1109-1122.

Van De Water, T.R., Lallemend, F., Eshraghi, A.A., Ahsan, S., He, J., Guzman, J., Polak, M., Malgrange, B., Lefebvre, P.P., Staecker, H. and Balkany, T.J. (2004): Caspases, the enemy within, and their role in oxidative stress-induced apoptosis of inner ear sensory cells. Otol. Neurotol., 25, 627-632.

van Ruijven, M.W., de Groot, J.C., Klis, S.F. and Smoorenburg, G.F. (2005): The cochlear targets of cisplatin: an electrophysiological and morphological time-sequence study. Hear Res. 205, 241-248. 